\title{
Factores de riesgo y complicaciones cardiovasculares en pacientes por alteraciones de fosforo $y$ calcio
}

\author{
Risk factors and cardiovascular complications in patients with phosphorus and calcium disorders
}

Factores de risco e complicações cardiovasculares em doentes com perturbações do fósforo e do cálcio

\section{Carolina Carolay Romero Merelo}

carolinarm_246@hotmail.com

https://orcid.org/0000-0002-3788-4247

\section{Luis Aarón Ríos Delgado}

luisaaronriosdelgado@gmail.com

https://orcid.org/0000-0003-0758-0020

\author{
David William Esteves Echanique \\ dawesteves@gmail.com \\ https://orcid.org/0000-0002-1203-801X
}

Karl Enrique Reyes Bravo

kenrike@gmail.com

https://orcid.org/0000-0002-6490-9569

\section{Universidad de Guayaquil, Guayaquil-Ecuador}

Recibido 6 de agosto 2021 / Arbitrado y aceptado 16 de septiembre 2021 / Publicado 14 de diciembre 2021

\begin{abstract}
RESUMEN
La enfermedad renal crónica es un problema de salud pública a nivel mundial y se define como una anormalidad patológica del riñón como hematuria o proteinuria, o una reducción de la tasa de filtrado glomerular menor a $60 \mathrm{ml}$-min por más de tres meses. En todos los estudios la prevalencia a esta enfermedad va aumentando debido a que sus principales causas diabetes e hipertensión se han convertido en pandemias. Objetivo. Demostrar los factores de riesgo y complicaciones cardiovasculares en pacientes por alteraciones minerales y óseas. Métodos. Se realizó un estudio descriptivo transversal en los pacientes del Instituto del riñón INRIDI San Martin de Enero a Nov del 2019, los métodos de investigación implementados fueron analítico y cualitativocuantitativo. El universo estuvo constituido por los 253 pacientes de la clínica de diálisis San Martin se obtuvo como muestra 70 pacientes basado en los criterios de exclusión. Resultados. Se halló que el $94.2 \%$ de los pacientes estudiados estuvieron en el grupo de casos sin hipercalcemia, así mismo $58.55 \%$ sentaron en el grupo con hiperfosfatemia. Entre los factores de riesgo se demostró la prevalencia en el sexo masculino, la hipertensión, diabetes y la dislipidemia. Conclusiones. La frecuencia de cambios en el metabolismo mineral y óseo en pacientes con ERC es muy variable, y este estudio revela aspectos epidemiológicos que permiten reconocer los problemas actuales de la enfermedad que viabilizaran reducir el riesgo de eventos cardiovasculares y mejorar la calidad de vida de los pacientes.
\end{abstract}

Palabras clave: ERC; Filtrado glomerular; Hipertensión

\begin{abstract}
Chronic kidney disease is a worldwide public health problem and is defined as a pathological abnormality of the kidney such as hematuria or proteinuria, or a reduction of the glomerular filtration rate to less than $60 \mathrm{ml}$-min for more than three months. In all studies the prevalence of this disease is increasing because its main causes diabetes and hypertension have become pandemics. Objective. To demonstrate the risk factors and cardiovascular complications in patients with mineral and bone alterations. Methods. A cross-sectional descriptive study was conducted in patients of the Instituto del riñón INRIDI San Martin from January to Nov 2019, the research methods implemented were analytical and qualitative-quantitative. The universe was constituted by the 253 patients of the San Martin dialysis clinic, 70 patients were obtained as a sample based on the exclusion criteria. Results: Results. It was found that $94.2 \%$ of the patients studied were in the group of cases without hypercalcemia, and $58.55 \%$ were in the group with hyperphosphatemia. Among the risk factors, the prevalence in the male sex, hypertension, diabetes and dyslipidemia was demonstrated. Conclusions. The frequency of changes in bone and mineral metabolism in patients with CKD is highly variable, and this study reveals epidemiological aspects that allow us to recognize the current problems of the disease that will make it feasible to reduce the risk of cardiovascular events and improve the quality of life of patients.
\end{abstract}

Key words: ERC; Glomerular filtration rate, Hypertension 


\section{RESUMO}

A doença renal crónica (CKD) é um problema de saúde pública mundial e é definida como uma anomalia patológica do rim, como a hematúria ou proteinúria, ou uma redução da taxa de filtração glomerular para menos de $60 \mathrm{ml}$-min durante mais de três meses. Em todos os estudos, a prevalência desta doença está a aumentar porque as suas principais causas de diabetes e hipertensão se tornaram pandemias. Objectivo. Demonstrar os factores de risco e as complicações cardiovasculares em doentes com doenças minerais e ósseas. Métodos. Foi realizado um estudo descritivo transversal em pacientes do instituto renal San Martin INRIDI de Janeiro a Novembro de 2019, os métodos de investigação implementados foram analíticos e qualitativos-quantitativos. O universo consistia em 253 pacientes da clínica de diálise de San Martin, 70 pacientes foram obtidos como amostra com base nos critérios de exclusão. Resultados. Verificou-se que $94,2 \%$ dos doentes estudados se encontravam no grupo de casos sem hipercalcemia, e 58,55\% estavam no grupo com hiperfosfatemia. Entre os factores de risco, foi demonstrada a prevalência de hipertensão, diabetes e dislipidemia no sexo masculino. Conclusões. A frequência das alterações do metabolismo ósseo e mineral em doentes com CKD é altamente variável, e este estudo revela aspectos epidemiológicos que nos permitem reconhecer os problemas actuais da doença que tornarão viável a redução do risco de eventos cardiovasculares e a melhoria da qualidade de vida dos doentes.

Palavras-Chave: ERC; Taxa de filtração glomerular; Hipertensão arterial

\section{INTRODUCCIÓN}

La enfermedad renal crónica, está asociada con un alto nivel de muerte y enfermedad cardiovascular. Las alteraciones minerales y óseas juegan un papel fundamental en la morbimortalidad de estos pacientes en personas con riñones saludables los niveles normales de calcio y fosforo se mantienen debido a un cuidadoso juego entre diversas hormonas que principalmente son tres: la hormona paratiroidea (PTH), calcitriol (un metabolito activo de la vitamina $D$ ) y factor de crecimiento de fibroblasto 23 , estas hormonas actúan principalmente en tres órganos diana: hueso, riñón e intestino $(1,2)$.
Es de observación y análisis que la ERC es una enfermedad emergente, considerando el hecho de que las dos principales etiologías son: diabetes mellitus e hipertensión arterial, enfermedades que van en aumento, así como ocurre con el envejecimiento poblacional. Dado que una de las complicaciones de la ERC es la enfermedad mineral ósea y que esta se relaciona con los eventos cardiovasculares, que es causa de muerte en los pacientes con ERC, se hace necesario conocer su fisiopatología para poder brindar una orientación a los pacientes que están en riesgo, a los que la padecen y saber referirlos oportunamente al médico especialista (3).

En todo paciente con enfermedad renal crónica sobre todo en las etapas más avanzadas encontramos; aumento del fosforo y parathormona, y disminución de la vitamina D y del calcio (4).

En este contexto, como punto de referencia es conveniente plantear que en el orden epidemiológico la Enfermedad renal crónica (ERC)esconsideradacomounproblemamundial de salud pública por su creciente prevalencia en las últimas décadas. Se ha demostrado que por cada paciente en un programa de diálisis o de trasplante puede haber un número mayor de casos con menor gravedad. Los mismos tienen un prominente riesgo cardiovascular y sufren una morbimortalidad por los eventos cardiovasculares, que podría tener una repercusión en la salud, mayor que el progreso hacia la necesidad de tratamiento sustitutivo de la función renal (5). 
Siguiendo la línea de pensamiento la visión epidemiológica actual de la ERC ha cambiado de manera notable. Restringida en sus inicios a una patología de incidencia baja como las enfermedades renales clásicas, hoy se encuentra bien documentado de que afecta a un porcentaje significativo de la población debido a que sus causas principales residen en los trastornos de alta prevalencia como el envejecimiento, la hipertensión arterial (HTA), la diabetes y la enfermedad vascular (6).

Revisiones realizadas de competentes autores exponen que la Enfermedad Renal Crónica se ha convertido en un problema de salud pública al ser una condición frecuente, afectando al $10 \%$ de la población adulta en todo el mundo, una de cada 10 personas sufre un grado de ERC y la Organización Mundial de la Salud (OMS) estima que hay 35 millones de muertes atribuibles a esta enfermedad. Según el estudio de la carga global de la enfermedad, realizado en el año 2010, la ERC ocupaba el puesto 27 en la lista de las causas del número total de muerte en el mundo en los años 90 y pasó a ocupar el puesto 18 de esta misma lista en el año 2010; la única patología que superó este cambio significativo en la lista fue el VIH/ Sida. La prevalencia de ERC en terapia de reemplazo renal en Latinoamérica aumentó de 119 pacientes por millón de población ( $\mathrm{pmp}$ ) en el año 1991 a 660 pacientes por millón de población (pmp) en 2010. De acuerdo con el último informe anual en los Estados Unidos de los pacientes con enfermedad renal crónica, la prevalencia aumentó en todos los países del 6 al 35\% durante el periodo desde 2006 hasta 2012 (7-9).
Tal como se ha visto la falta de reconocimiento precoz de ERC tiene consecuencias negativas. La declinación de la función renal se asocia directamente a la acumulación de complicaciones con pronóstico adverso. Durante su evolución silenciosa con ERC, el paciente experimenta deterioro progresivo renal y morbi-mortalidad cardiovascular. Estudios recientes muestran que, la probabilidad de fallecimiento de pacientes con ERC por complicaciones cardiovasculares, es mucho mayor que la progresión por falla renal terminal. Si la ERC y sus factores de riesgo no son detectados, se pierden oportunidades únicas de prevención y tratamiento (10).

Considerando, que la enfermedad renal con frecuencia comienza en una etapa tardía de la vida y que progresa en forma lenta, la mayoría de las personas con una velocidad de filtración glomerular reducida, muere de una enfermedad cardiovascular antes de desarrollar una IRC terminal $(11,12)$.

La ECV, como complicación de la ERC, merece una consideración especial por los siguientes motivos: Los eventos CV son más frecuentes que la falla renal en personas con ERC; la ECR es, con alta probabilidad, un factor de riesgo CV, y la ECV, en personas con ECR, tiene tratamiento y es potencialmente prevenible $(13,14)$.

La repercusión de la ECR sobre la mortalidad se ha incrementado a nivel mundial; destacando el impacto que tiene sobre los años de vida potencial perdidos, estimados en la actualidad en alrededor de 13,75 años perdidos en el año 2013 y 13,71 años para el 2014 respectivamente $(15,16)$. 
En Ecuador, la ERC es un importante problema de salud pública, por su alta prevalencia. Se estima que afecta al $11 \%$ de la población adulta, como todos los procesos crónicos, produce un elevado gasto sanitario, condicionado por una alta tasa de morbilidad, y un importante consumo de recursos farmacológicos. Diferentes estudios poblacionales han demostrado que, la tasa de mortalidad global disminuye de forma significativa, cuando se realiza un diagnóstico precoz de las alteraciones hemodinámicas, minerales y hormonales (10).

Sus principales factores de riesgo son la diabetes y la hipertensión arterial (HTA). Estos forman parte del grupo de enfermedades crónicas, las que han aumentado sostenidamente su prevalencia e incidencia. Ambas constituyen hoy un problema de salud pública devastador, debido a las consecuencias médicas, sociales y económicas, para los pacientes, sus familias, y los sistemas de salud.

Es saludable acotar que la ECR es la cuarta causa de mortalidad general en el Ecuador y una de las más costosas. El costo de una hemodiálisis alcanza, por ejemplo, USD 300 y una hemofiltración, USD 600. En total una persona necesitaría entre USD 4200 y 8400 cada seis semanas.

Para este fin el Instituto de riñón y diálisis San Martin cordialmente abre sus puertas y permite el desarrollo de esta investigación y la obtención de resultados que puedan ser aplicados para ofrecer un mejor servicio cambiando para bien la vida de sus pacientes.

\section{MATERIALES Y MÉTODOS}

Se realizó un estudio descriptivo, transversa en los pacientes en el Instituto del riñón INRIDI San Martin de Enero a Nov del 2019. Con la finalidad de demostrar los factores de riesgo y complicaciones cardiovasculares en pacientes por alteraciones de fosforo y calcio, mediante la observación indirecta contribuyendo a los estudios de la institución evitando de esta forma tasa más altas de mortalidad de este padecimiento. Los métodos de investigación utilizados fueron analítico y cualitativocuantitativo. El universo estuvo constituido por los 253 pacientes de la clínica de diálisis San Martin Según la fórmula $n=N / e^{2}(N-$ 1) +1 se obtuvo como muestra 70 pacientes basado en los criterios de exclusión lo que corresponde al $27.66 \%$ del universo de estudio. Con relación a la operacionalización de las variables se tuvo en cuenta como variable independiente Alteraciones de fosforo y calcio y como variables dependientes: Enfermedad renal crónica y Estadio de ERC. Como variables intervinientes: sexo, edad, procedencia, raza, factores de riesgo y tiempo de la evolución de la enfermedad. La recolección de datos fue mediante la revisión de documentos y registros de las carpetas y datos de los pacientes. La totalidad de los datos fueron procesados de forma automatizada. Se utilizó los programas de recolección de datos SPSS, Excel. 


\section{RESULTADOS}

Se exponen:

En la serie primaron (Tabla 1 ) $5.80 \%$ con hipercalcemia, definida como valores séricos de calcio mayores a $10.5 \mathrm{mg} / \mathrm{dl}$. Teniendo en cuenta que estos pacientes tienen una tasa de filtrado glomerular menor a $15 \mathrm{ml} / \mathrm{min} / 1.73$ $\mathrm{m} 2$. Este resultado divide al total de pacientes en dos grupos.

- Con enfermedad renal crónica con hipercalcemia

- Con enfermedad renal crónica sin hipercalcemia

Tabla 1. Pacientes con ERC que tienen hipercalcemia.

\begin{tabular}{lcc}
\hline \multicolumn{1}{c}{ Pacientes con ERC } & Número & Porcentaje \\
\hline Grupo de casos con hipercalcemia & 4 & $5,80 \%$ \\
Grupo de casos sin hipercalcemia & 66 & $94.2 \%$ \\
\hline TOTAL & $\mathbf{7 0}$ & $\mathbf{1 0 0 \%}$ \\
\hline
\end{tabular}

FUENTE: Instituto del riñon y diálisis “INRIDI “ San Martin.

Como se observa en la Tabla 2, el total de pacientes del estudio (70) se reporta un $58.55 \%$ con hiperfosfatemia, definida como valores séricos de calcio mayores a $4.6 \mathrm{mg} / \mathrm{dl}$. Teniendo en cuenta que estos pacientes tienen una tasa de filtrado glomerular menor a $15 \mathrm{ml}$ $\min / 1.73 \mathrm{~m} 2$. Este resultado divide al total de pacientes en dos grupos:

- Con enfermedad renal crónica con hiperfosfatemia

- Con enfermedad renal crónica sin hiperfosfatemia

Tabla 2. Pacientes con ERC que tienen hipercalcemia.

\begin{tabular}{lcc}
\hline \multicolumn{1}{c}{ Pacientes con ERC } & Número & Porcentaje \\
\hline Grupo de casos con hiperfosfatemia & 41 & $58,55 \%$ \\
Grupo de casos sin hiperfosfatemia & 29 & $41.43 \%$ \\
\hline TOTAL & $\mathbf{7 0}$ & $\mathbf{1 0 0 \%}$ \\
\hline
\end{tabular}




\section{ERC hiperfosfatemia}

Véase en la Tabla 3, el grupo etario de entre 20 y 40 años los 4 pacientes que corresponden a este grupo no presentaron hipercalcemia lo que corresponde a un $5.71 \%$ de la muestra; sin hipercalcemia. El siguiente grupo etario de entre 41 a 61 años presento 3 pacientes con hipercalcemia lo que corresponde al $4.28 \%$ y 36 pacientes sin hipercalcemia lo que corresponde al $51.4 \%$ teniendo como total de este grupo etario 55.68\%, el último grupo etario > 60 años presento un caso de hipercalcemia las cuales corresponden al $1.43 \%$ y 26 casos negativos lo que corresponde al $37.14 \%$ definida como valores séricos de calcio mayores a $10.5 \mathrm{mg} / \mathrm{dl}$.
Teniendo en cuenta que estos pacientes tienen una tasa de filtrado glomerular menor a $15 \mathrm{ml}$ $\min / 1.73 \mathrm{~m} 2$.

De los 70 pacientes con enfermedad renal crónica y alteración del calcio, el $5.71 \%$ de los pacientes (4) tenía menos de 40 años y el resto 66 pacientes tenía más de 40 . El análisis demostró que existe asociación estadísticamente significativa entre la edad y el desarrollo de alteración de calcio en los pacientes con ERC $(p=0,7821)$. La estimación de riesgo demuestra que tener más de 40 años eleva el riesgo 1.54 veces de desarrollar alteraciones de calcio en ERC (OR: 1.5432 IC 95\%:0.0713-33.3909).

Tabla 3. Relación entre la edad e hipercalcemia en ERC.

\begin{tabular}{ccccc}
\hline \multirow{2}{*}{ Variables demográficas } & \multicolumn{2}{c}{ Hipercalcemia } & TOTAL \\
\hline Grupos de edades & $\mathbf{S i}$ & $\mathbf{N o}$ & 4 \\
& $\mathbf{2 0 - 4 0}$ & 0 & 4 & $5,71 \%$ \\
& $\mathbf{4 1 - 6 0}$ & 0 & $3,71 \%$ & 39 \\
& & 36 & $55,68 \%$ \\
TOTAL & $\mathbf{6 0}$ & 1 & $51,4 \%$ & 27 \\
& & $26 \%$ & $37,14 \%$ & $38,57 \%$ \\
& & $4,43 \%$ & 66 & 70 \\
\hline
\end{tabular}

En la abla 4 queda reflejado que el grupo etario de entre 20 y 40 años los 4 pacientes que corresponden a este grupo dos pacientes presentan hiperfosfatemia $(2.85 \%)$ y dos no presentan hiperfosfatemia (2.85\%) lo que corresponde a un $5.71 \%$ de la muestra; sin hipercalcemia. El siguiente grupo etario de entre 41 a 61 años presento 18 pacientes con hiperfosfatemia lo que corresponde al $25.71 \%$ y 21 pacientes sin hiperfosfatemia lo que corresponde al $30 \%$ teniendo como total de este grupo etario $55.68 \%$, el último grupo etario $>60$ años presento 21 casos de hiperfosfatemia las cuales corresponden al $30 \%$ y 6 casos negativos lo que corresponde al $8.57 \%$, definida como valores séricos de calcio 
mayores a $10.5 \mathrm{mg} / \mathrm{dl}$. Teniendo en cuenta que estos pacientes tienen una tasa de filtrado glomerular menor a $15 \mathrm{ml} / \mathrm{min} / 1.73 \mathrm{~m} 2$.

De los 70 pacientes con enfermedad renal crónica y alteración del calcio, el $5.71 \%$ de los pacientes (4) tenía menos de 40 años y el resto $66(74.29 \%)$ pacientes tenía más de 40. El análisis demostró que existe asociación estadísticamente significativa entre la edad y el desarrollo de alteración de fosfato en los pacientes con ERC $(p=0,7213)$. La estimación de riesgo demuestra que tener menos de 40 años tiene un ODDS RATIO 0.6923 lo cual se interpreta como un factor de protección de desarrollar alteraciones de fosforo en ERC (OR: 0.6923 IC 0.0918-5.2214).

Tabla 4. Relación entre la edad e hipercalcemia en ERC.

\begin{tabular}{|c|c|c|c|c|}
\hline \multirow{2}{*}{\multicolumn{2}{|c|}{ Variables demográficas }} & \multicolumn{2}{|c|}{ Hiperfosfatemia } & \multirow{3}{*}{$\begin{array}{c}\text { TOTAL } \\
4\end{array}$} \\
\hline & & \multirow{2}{*}{$\frac{\mathbf{S i}}{2}$} & \multirow{2}{*}{$\frac{\text { No }}{2}$} & \\
\hline Grupos de edades & $20-40$ & & & \\
\hline & & 2,85 & $2,85 \%$ & $5,71 \%$ \\
\hline & $41-60$ & 18 & 21 & 39 \\
\hline & & $25,71 \%$ & $30 \%$ & $55,71 \%$ \\
\hline & $\geq 60$ & 21 & 6 & 27 \\
\hline & & $30 \%$ & $8,57 \%$ & $38,57 \%$ \\
\hline \multirow[t]{2}{*}{ TOTAL } & & 41 & 29 & 70 \\
\hline & & $58,56 \%$ & $41,44 \%$ & $100 \%$ \\
\hline
\end{tabular}

Para la identificación de los factores de riesgo en pacientes por alteraciones de fosforo y calcio se tomó en cuenta el género y las enfermedades crónicas existente se muestra en la Tabla 5 el 42 (60\%) pacientes de género masculino, $28(40 \%)$ pacientes de género femenino, pacientes mayores de 50 años (71.4\%), 28 (40\%) pacientes con hipertensión, $33(47.1 \%)$ pacientes con diabetes y $17(24.28 \%)$ pacientes con dislipidemia (Tabla 5, Gráfico 5).

Tabla 5. Factores de riesgo en pacientes por alteraciones de fosforo y calcio.

\begin{tabular}{|c|c|c|}
\hline Variables & Número & Porcentaje \\
\hline Hombres & 42 & $60 \%$ \\
\hline Mujeres & 28 & $40 \%$ \\
\hline Mayores de 50 & 50 & $71.4 \%$ \\
\hline Hipertensión & 28 & $40 \%$ \\
\hline Diabetes & 33 & $47.1 \%$ \\
\hline Dislipidemia & 17 & $24.28 \%$ \\
\hline
\end{tabular}


Se observa en la Tabla 6 las alteraciones encontradas en la PTH en los pacientes estudiados. Un paciente (1.43\%) con PTH > 450, lo que se asocia a enfermedad ósea de alto remodelado; y se encontraron 62 pacientes $88.57 \%$ lo que corresponde a enfermedad ósea de bajo remodelado.

Tabla 6. Alteraciones encontradas en la PTH en los pacientes estudiados.

\begin{tabular}{lcc}
\hline \multicolumn{1}{c}{ Pacientes con ERC } & Frecuencia & Porcentaje \\
\hline PTH elevada $>\mathbf{4 5 0}$ & 1 & $1.43 \%$ \\
PTH normal & 7 & $10,00 \%$ \\
\hline PTH disminuida $<\mathbf{1 5 0}$ & 62 & $\mathbf{8 8 . 5 7 \%}$ \\
\hline Total & $\mathbf{7 0}$ & $\mathbf{1 0 0 , 0 0 \%}$ \\
\hline
\end{tabular}

\section{DISCUSIÓN}

Ha sido registrada la ERC como un importante factor de riesgo, independiente de varios resultados adversos para la salud, incluyendo en primer lugar a las enfermedades cardiovasculares. Es considerada, un problema de salud pública de gran relevancia por su infra diagnóstico, su morbimortalidad vascular y su prevalencia, sobre todo, en diabéticos e hipertensos (17).

Uno de los grandes retos de la nefrología son las alteraciones del metabolismo mineral y óseo (MMO), lo cual están constante renovación y de gran complejidad, esto lo sustenta las diferentes estrategias terapéuticas, guías clínicas y objetivos que se han ido planteando a lo largo de los años (18).

Entre los cambios más importantes en este terreno es el reconocimiento, relativamente reciente, de que las alteraciones del metabolismo óseo-mineral relacionadas con la enfermedad renal crónica (ERC) van más allá del hueso, y se consideran un trastorno sistémico, que incluyen el sistema cardiovascular, las cuales tienen un gran impacto sobre la morbimortalidad de los pacientes con ERC, y otro aspecto importante es conocer que estos trastornos se inician en los primeros estadios de la ERC, mucho antes de que se produzca una reducción grave del filtrado glomerular o de que se establezca la necesidad de terapia renal sustitutiva (18).

En las revisiones realizadas; varios autores alistan la existencia de una serie de trabajos alrededor del mundo de CKD-MBD los cuales cada uno le da un enfoque distinto en las diversas poblaciones como el trabajo de Rroji M. et al sobre si se debe considerar el sistema cardiovascular en pacientes con ERC, en el que demuestra la alta incidencia de enfermedad cardiovascular en pacientes con ERC, esto es igual en Ecuador cerca del $50 \%$ de pacientes son hipertensos o tienen alguna otra afección cardiovascular y este es un factor de riesgo de suma importancia de necesitar 
terapia sustitutiva de la función renal, también demostró que la presencia de CKD- MBD en los pacientes con ERC aumentaba el riesgo de eventos vasculares y la mortalidad asunto que no es tratado en este trabajo pero sin duda, se deja claro que es la principal causa de muerte en estos pacientes. También se describe como esta enfermedad no es de origen ateromatoso lo que se descubrió al no tener resultados utilizando drogas que disminuyen la progresión de las dislipidemias y se describe los nuevos biomarcadores.

Así mismo, Chao et al., describe la osteodistrofia renal en pacientes con nefropatía diabética, la cual es otra de las principales causas de ERC; sobre la relación de la aparición de estas complicaciones con la presencia de nefropatía diabética.

Ugarte F, Carranza en su estudio, consideraron que el desarrollo de la nefropatía diabética se inicia en los primeros años posteriores al inicio de la diabetes, existiendo elementos de laboratorio que permitirían la detección de factores de riesgo y su diagnóstico precoz y, lo que es de especial interés, posibles tratamientos que permitirian retardar o prevenir el desarrollo de ella. En nuestro estudio se revelo un número no despreciable de pacientes diabéticos (19).

Existen algunos que ejemplifican mejor los resultados como el de Cohen-Solal $M$, FunchBrentano T, Urena P sobre la CKD-MBD y la presencia de fragilidad ósea (20).

Siguiendo la línea de pensamiento Allen, Swallow, y Metzger, intenta cambiar la forma en la que se percibe la enfermedad renal y ósea y la salud esquelética.
Lioufas et al. Pedagogos, Hawley et al. describen las calcificaciones árticas y la rigidez arterial como factor de predicción de la sobrevida, basados en que resultado tendría la reducción de los niveles de fosfato utilizando el carbonato de lantano como nació el estudio IMPROVE-CKD en el cual se medía la velocidad de onda de pulso carotidea y femoral y se utilizaba el índice de agatson (21).

En cuanto a trabajos de pregrado, existe un trabajo de la Dra. Johanna Puruncajas sobre este mismo tema hecho para la Pontificia Universidad Católica del Ecuador con un enfoque diferente centrado en el hiperparatiroidismo secundario. En el cual al igual que en este trabajo se habla de la gran afección cardiovascular de estos pacientes y la elevada mortalidad (22).

\section{CONCLUSIONES}

La frecuencia de cambios en el metabolismo mineral y óseo en pacientes con ERC es muy variable, y este estudio revela aspectos epidemiológicos que permiten reconocer los problemas actuales de la enfermedad. Reconociendo los esfuerzos de la directiva para realizar los cambios necesarios en el sistema de salud para administrar mejor el tratamiento, reducir el riesgo de eventos cardiovasculares y mejorar la calidad de vida de los pacientes. 


\section{REFERENCIAS BIBLIOGRÁFICAS}

1. Loaiza J, Condori M, Quispe G, Pinares M, Cruz A, Atamari N. Mortalidad y factores asociados en pacientes con enfermedad renal crónica en hemodiálisis en un hospital peruano. Rev haban cienc méd [Internet]. 2019;18(1):16475. Disponible en: http://scielo.sld.cu/ scielo.php?script=sci_arttext\&pid=S1729519X2019000100164\&lng=es

2. Francis E, Allen A, Herrera P, Kuo C, Cardenas $M$, Feldman $\mathrm{H}$, et al. Establishing a higher priority for chronic kidney disease in Peru. Lancet Glob Heal. 2016;4(1):17-8

3. Bernuy J, Gonzales G. Metabolismo mineral óseo en pacientes con enfermedad renal crónica: revisión sobre su fisiopatología y morbimortali-dad. Rev Peru Med Exp Salud Publica. 2015;32(2):326-34.

4. Trujillo $\mathrm{H}$, Sierra J, Osorno L. Alteraciones del metabolismo mineral y óseo asociadas a la enfermedad renal crónica. Rev Med MD [Internet]. 2015;6(2):151.160. Disponible en: https://www.imbiomed.com.mx/articulo. php?id=105112

5. Stevens L, Schmid C, Greene T, Zhang Y, Beck $\mathrm{G}$, Froissart $\mathrm{M}$, et al. Comparative performance of the CKD Epidemiology Collaboration (CKDEPI) and the Modification of Diet in Renal Disease (MDRD) Study equations for estimating GFR levels above $60 \mathrm{~mL} / \mathrm{min} / 1.73 \mathrm{~m} 2$. Am J Kidney Dis. 2010;56(3):486-95.

6. Souchay L, Sotolongo D, Álvarez Y, Castillo M. Complicaciones cardiovasculares y sus factores de riesgo en pacientes adultos portadores de Enfermedad Renal Crónica. Rev Panorama Cuba y Salud [Internet]. 2019;14(2):310. Disponible en: http://www.revpanorama. sld.cu/index.php/rpan/article/view/

7. Mitchell C. OPS OMS | La OPS/OMS y la Sociedad Latinoamericana de Nefrología llaman a prevenir la enfermedad renal y a mejorar el acceso al tratamiento. Pan American Health Organization / World Health Organization. 2015. p. 1-5.

8. Gonzalez M, Rosa G, Pecoits R, Ferreiro A, G $G$, Cusumano A. Burden of disease: prevalence and incidence of ESRD in Latin America. Clin Nephrol [Internet]. 2015;83(7 Suppl 1):3-6. Disponible en: http://www.ncbi.nlm.nih.gov/ pub $\neg$ med/25725232

9. Afsar B, Turkmen K, Covic A, Kanbay M. An update on coronary artery disease and chronic kidney disease. Int J Nephrol. 2014;2014.

10. Díaz M, Gómez B, Robalino M, Lucero $S$. Comportamiento epidemiológico en pacientes con enfermedad renal crónica terminal en Ecuador. CCM [Internet]. 2018;22(2):31224. Disponible en: http://scielo.sld.cu/ scielo.php?script $=$ sci_arttext\&pid $=\mathrm{S} 1560$ $43812018000200011 \& \mathrm{lng}=$ es . 24 . Disponible en: http://scielo.sld.cu/scielo. php?script=sci_arttext\&pid=S1560$43812018000200011 \& \operatorname{lng}=\mathrm{es}$.

11. Alcázar AR, Orte L, González E, Górriz JL, Navarro JF, Martín de Francisco, A. L., Egocheaga $\mathrm{MI}$, et al. Documento de consenso SENsemFYC sobre la enfermedad renal crónica. Nefrologia [Internet]. 2013;28(3):273-82. Disponible en: https://www.revistanefrologia. com / es-documento-consenso-sensemfyc-sobre-enfermedad-renal-cronicaarticulo-X0211699508005614

12. Martínez A, Górriz JL, Bover J, Segura-de la Morena J, Cebollada J, Escalada J, et al. Documento de Consenso para la detección y manejo de la enfermedad renal crónica. Nefrologia [Internet]. 2014;34(2):243-62. Disponible en: https://scielo.isciii.es/pdf/ nefrologia/v34n2/documento_consenso.pdf $\% 0 A \% 0 A$

13. Husseini N, Kaskar O, Goldstein L. Chronic kidney disease and stroke. Adv Chronic Kidney Dis. 2014;21(6):500-8. 
14. Ricardo A, Lash J, Fischer M, Lora C, Budoff M, Keane $M$, et al. Cardiovascular disease among hispanics and non-hispanics in the chronic renal insufficiency cohort (CRIC) study. Clin J Am Soc Nephrol [Internet]. 2011;6(9):2121-3. Disponible en: 10.2215/CJN.11341210

15. Pérez JL, Herrera R, Almaguer R. Situación de la enfermedad renal crónica en Cuba. En: Anuario Cuba Nefro-Red. La Habana; 2014.

16. Medellín J, Carrillo G. Soporte social percibido y calidad de vida de personas con enfermedad renal crónica sometidas a trasplante renal. Rev Av en Enfermería. 2014;32(3):206-16.

17. Sánchez-Celaya M, Tranche S. Documento de consenso sobre la enfermedad renal crónica. S.E.N-semFYC. Atención Primaria. 2014;46:453-4.

18. Dolores M. Alteraciones del Metabolismo Mineral: Calcio, Fósforo, PTH, Vitamina D, FGF-23, Klotho. Nefrología al Día [Internet]. 2020; Disponible en: https://www. nefrologiaaldia.org/es-articulo-alteracionesdel-metabolismo-mineral-calcio-311

19. Chia-Ter,Szu-Ying Lee,Wei-Shun Yang, Chung-Yen,Chih-Kang Chiang,Jenq-Wen Huang, Kuan-Yu Hung.Serum vitamin D levels are positively associated with variccella

\section{ACERCA DE LOS AUTORES}

Carolina Carolay Romero Merelo. Médico, Universidad de Guayaquil. Internado Rotativo, Hospital Universitario de Guayaquil y Hospital de Infectología. Experiencia en Soporte Vital Cardiovascular Avanzado, Soporte Vital Básico. Universidad de Guayaquil, Ecuador.

David William Esteves Echanique. Médico, Universidad de Guayaquil. Internado Rotativo, Hospital Universitario de Guayaquil y Hospital de Infectología. Ayudante de Cátedra de Nefrología. Experiencia en Soporte Vital Cardiovascular Avanzado, Soporte Vital Básico. Universidad de Guayaquil, Ecuador.

Luis Aarón Ríos Delgado. Médico, Universidad de Guayaquil. Internado Rotativo, Hospital León Becerra de Milagro. Experiencia en Soporte Vital Cardiovascular Avanzado. Medicatura Rural en el Centro de Salud Simiatug- Provincia Bolívar. Universidad de Guayaquil, Ecuador.

Karl Enrique Reyes Bravo. Médico, Universidad Estatal de Guayaquil. Internado rotativo en el Hospital General Sagrado Corazón de Jesús, Quevedo. Medicatura Rural en el centro de salud Simiatug perteneciente a la provincia de Bolívar. Universidad de Guayaquil, Ecuador. zoster inmunity in chronic dialysis patients. Sci Rep.2014 Dec 9;4:7371.doi:10.1038/srep07371.

20. Ugarte F, Carranza C. Nefropatía Diabética. Rev Chil pediatría [Internet]. 2002;73(5):45560. Disponible en: http://www.scielo.cl/ scielo.php?script=sci_arttext\&pid=S0370$41062002000500002 \& \operatorname{lng}=\mathrm{es}$

21. Cohen M, Funch T, Urena P. Bone fragility in patients with chronic kidney diseases. Endocr Connect. 2020;9(4):93-101.

22. Lioufas N, Sm Chen S, Hawley C, Pedagogos $E$, Pascoe $E, A V$, et al. Effect of phosphatelowering therapy on cardiac structure and function-a sub-study of the improve-CKD trial. Nephrology (Carlton). 2020;25(SUPPL 3).

23. Puruncajas J. Prevalencia de hiperparatiroidismo secundario a enfermedad renal crónica estadio cinco en pacientes bajo tratamiento de hemodiálisis en el centro de hemodiálisis vyr durante el 2017 [Internet]. Pontificia Universidad Catolica del Ecuador; 2018. Disponible en: http://repositorio.puce. edu.ec/handle/22000/15398

Conflicto de intereses: Ninguno declarado por los autores.

Financiación: Ninguna declarada por los autores.

Agradecimiento: Ninguno manifestado por los autores 